\title{
Pseudo Polynomial Size LP Formulation for Calculating the Least Core Value of Weighted Voting Games*
}

\author{
Tokyo Institute of Technology Masato Tanaka \\ Tokyo Institute of Technology Tomomi Matsui
}

August 24, 2021

\begin{abstract}
In this paper, we propose a pseudo polynomial size LP formulation for finding a payoff vector in the least core of a weighted voting game. The numbers of variables and constraints in our formulation are both bounded by $\mathrm{O}\left(n W_{+}\right)$, where $n$ is the number of players and $W_{+}$is the total sum of (integer) voting weights. When we employ our formulation, a commercial LP solver calculates a payoff vector in the least core of practical weighted voting games in a few seconds. We also extend our approach to vector weighted voting games.
\end{abstract}

Keywords: Weighted voting games; least core; linear programming JEL classification: C61, C63, C71

\section{Introduction}

This paper deals with a special class of simple games, called weighted voting games, which constitute a familiar example of voting systems. In a weighted

${ }^{*}$ A preliminary version of this paper was presented at the 12th Annual Meeting of the Asian Association for Algorithms and Computation, April 19 - 21, 2019, Seoul, South Korea. This work was supported by JSPS KAKENHI Grant Numbers JP26285045, JP26242027, JP20K04973. We thank Kento Tanaka for extensive discussions. 
voting game, each player has a voting weight (intuitively corresponding to their contribution), and a coalition wins if the sum of its members' weights meets or exceeds a given threshold.

Over the years, many power indices of voting games have been proposed and studied, such as the Shapley-Shubik index [1], the Banzhaf index [2], and the Deegan-Packel index [3]. The problem of computing the players' power indices has received ample attention (see [4] for example) and its computational complexity is well-understood [5, 6, 7, 8, ,9].

The focus of this work is on the solution concept called the least core, proposed by Maschler, Peleg, and Shapley [10], which is a natural relaxation of the core. Elkind et al. [6] showed some intractability results including coNP-hardness for checking the non-emptyness of the $\varepsilon$-core of a weighted voting game. They proposed a pseudo polynomial time algorithm to compute a payoff vector in the least core, which is based on the (polynomial time) ellipsoid method and a pseudo polynomial time separation oracle. They also proposed a fully polynomial time approximation scheme (FPTAS) for the value of the least core. The nucleolus is a unique payoff vector with a lexicographically maximal excess vector, which is contained in the least core (e.g., see [11, 6]). Pseudo-polynomial time algorithms for finding the nucleolus of a weighted voting game are proposed in [12, 13].

In this paper, we propose a pseudo-polynomial size LP "formulation" for finding a payoff vector in the least core of a weighted voting game. The numbers of variables and constraints of our formulation are both bounded by $\mathrm{O}\left(n W_{+}\right)$, where $n$ is the number of players and $W_{+}$is the sum of (integer) voting weights. Thus, a polynomial time algorithm for general linear programming problems finds a payoff vector in the least core in pseudo polynomial time. An advantage of our approach is that one can adopt his/her favored software (LP solver) for calculating a payoff vector in the least core. The computational experiment shows the effectiveness and efficiency of the proposed formulation. In the last section, we also extend our approach to vector weighted voting games.

\section{Notations and Definitions}

Let $N=\{1,2, \ldots, n\}$ be a set of players. A weighted voting game $G$ is defined by a sequence of positive integers $G=\left[q ; w_{1}, w_{2}, \ldots, w_{n}\right]$, where we may think of $w_{i}$ as the number of votes of player $i$ and $q$ as the quota needed for a 
coalition to win. In this paper, we assume that $0<q \leq w_{1}+w_{2}+\cdots+w_{n}$. A coalition $S \subseteq N$ is called a winning coalition when the inequality $q \leq \sum_{i \in S} w_{i}$ holds. The set of all the winning coalitions is denoted by $\mathcal{W}$. A weighted voting game gives a simple game $(N, v)$ defined by a characteristic function $v: 2^{N} \rightarrow\{0,1\}$ ensuring that $v(S)=1$ if and only if $S \in \mathcal{W}$.

In this paper, we discuss a solution concept, called the least core, of a characteristic function game. Given a characteristic function game $(N, v)$, a pre-imputation of $(N, v)$ is a non-negative payoff vector $\boldsymbol{x}=\left(x_{i} \mid i \in N\right)$ such that $\sum_{i \in N} x_{i}=v(N)$. The core of $(N, v)$ is the set of pre-imputations satisfying $\sum_{i \in S} x_{i} \geq v(S)$ for all $S \subseteq N$. The $\varepsilon$-core of $(N, v)$ is the set of pre-imputations satisfying $\sum_{i \in S} x_{i} \geq v(S)-\varepsilon, \forall S \subseteq N$. The least core of $(N, v)$ is its $\varepsilon^{*}$-core where $\varepsilon^{*}=\inf \{\varepsilon \mid \varepsilon$-core of $(N, v)$ is non-empty $\}$.

It is easy to see that the least core of a weighted voting game is a set of vectors $\boldsymbol{x} \in \mathbb{R}^{N}$ which confirm that $\left(\varepsilon^{*}, \boldsymbol{x}\right)$ is optimal to the following linear programming problem:

$$
\begin{aligned}
\text { P1: } \min . & \varepsilon \\
\text { s.t. } & \sum_{i \in S} x_{i} \geq 1-\varepsilon \quad(\forall S \in \mathcal{W}), \\
& \sum_{i \in N} x_{i}=1, \\
& x_{i} \geq 0
\end{aligned} \quad(\forall i \in N),
$$

where $\varepsilon^{*}$ is the optimal value of P1 (see [14, 6]). Here we note that the number of constraints of P1 may be exponential in $n$.

One of the most popular methods to solve linear programming problems is the simplex method designed by Dantzig [15]. It is quite efficient in practice and on average, but no polynomial-time worst-case running time bound has been proved. The ellipsoid method is the first polynomial-time method for linear programming given by Khachiyan [16]. The ellipsoid method is not competitive with the simplex method in practice (e.g., see [17]) but it has important theoretical side-effects. Specifically, the ellipsoid method does not require listing all constraints of an LP problem a priori, but allows that they are generated when needed. A separation oracle for a linear (feasibility) program is a procedure that, given a candidate solution, determines whether it is feasible and, if not, outputs a violated constraint. If a linear program admits a separation oracle, then an optimal solution can be found by the ellipsoid method (see [18] for a detailed discussion). In 1984, Karmarkar [19] developed a method for linear programming called Karmarkar's algorithm, which runs in polynomial time. Karmarkar's algorithm falls within the class 
of interior point methods characterized by polynomial complexity. Moreover, they have efficient implementations, competing with the simplex method.

When we solve P1 directly, we need to generate all the (minimal) winning coalitions growing exponentially in $n$. Elkind et al. [6] adopted the ellipsoid method and proposed a pseudo polynomial time separation oracle, which yields a pseudo polynomial time algorithm for solving $\mathrm{P} 1$.

\section{Formulation}

In this section, we propose a new formulation for calculating a payoff vector in the least core of a weighted voting game. For any positive integer $\alpha,[\alpha]$ and $[\alpha]_{0}$ denote the set of integers $\{1,2, \ldots, \alpha\}$ and $\{0,1, \ldots, \alpha\}$, respectively. Here we note that the set of players $N$ is equal to $[n]$. We define that $W_{+}=\sum_{i=1}^{n} w_{i}$. We introduce an acyclic directed graph $\Gamma=(V, A)$ with a vertex set $V=[n]_{0} \times\left[W_{+}\right]_{0}$ and an arc set $A=A_{0} \cup A_{1} \cup \cdots \cup A_{n}$ defined by

$$
\begin{aligned}
& A_{0}=\left\{((i-1, \alpha),(i, \alpha)) \mid(i, \alpha) \in[n] \times\left[W_{+}\right]_{0}\right\}, \\
& A_{i}=\left\{\left((i-1, \alpha),\left(i, \alpha+w_{i}\right)\right) \mid \forall \alpha \in\left[W_{+}-w_{i}\right]_{0}\right\} \quad(\forall i \in N=[n]) .
\end{aligned}
$$

We define a specified set of vertices $T=\{(n, \alpha) \subseteq V \mid q \leq \alpha\}$. The vertex $(0,0) \in V$ is called the source and denoted by $s$. Figure 1 shows an example of $\Gamma$. A directed path in $\Gamma$ from the source $s=(0,0)$ to a vertex in $T$ is called an $s-T$ path. It is easy to see that there exists a bijection between $\mathcal{W}$ and a set of $s-T$ paths.

Given a vector $\boldsymbol{x} \in \mathbb{R}^{N}$, we introduce an arc length function $w^{\boldsymbol{x}}: A \rightarrow \mathbb{R}$ defined by

$$
w^{\boldsymbol{x}}(a)= \begin{cases}0 & \left(\text { if } a \in A_{0}\right) \\ x_{i} & \left(\text { if } a \in A_{i}\right)\end{cases}
$$

Then, it is easy to show that a pair $(\varepsilon, \boldsymbol{x})$ satisfies $\sum_{i \in S} x_{i} \geq 1-\varepsilon(\forall S \in \mathcal{W})$ if and only if the length (defined by $w^{\boldsymbol{x}}$ ) of the shortest $s-T$ path is greater than or equal to $1-\varepsilon$. Given a pre-imputation $\boldsymbol{x}$, the length of the shortest $s$-T path, denoted by $1-\varepsilon^{\prime}$, satisfies $\varepsilon^{\prime}=\inf \{\varepsilon \mid \varepsilon$-core of $(N, v)$ includes $\boldsymbol{x}\}$. Thus, the problem of calculating the least core value corresponds to a problem of finding a pre-imputation $\boldsymbol{x}$ which maximizes the shortest $s-T$ path length. Here we note that we can transform the shortest $s-T$ path problem to an ordinary 2-terminal shortest path problem by contracting the set of vertices $T$ to an artificial terminal vertex. 


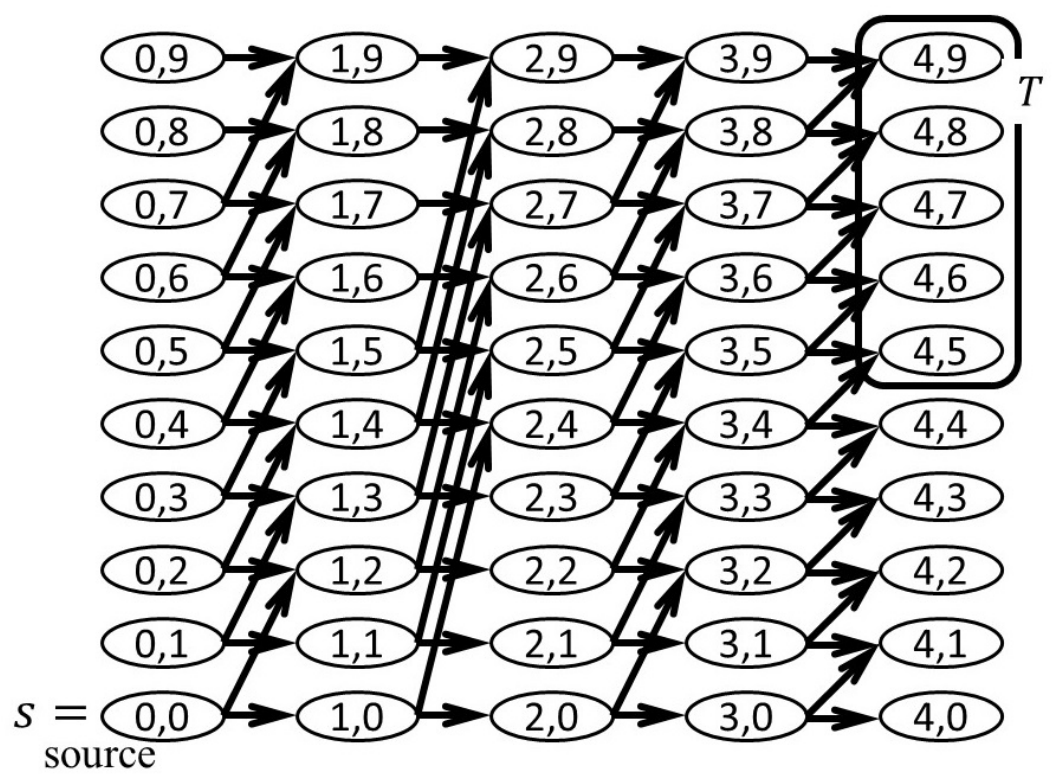

Figure 1: Directed graph $\Gamma$ corresponding to $G=[5 ; 2,4,2,1]$.

In the following part, we employ a "dual" linear programming formulation for the shortest path problem. An ordinary primal linear programming formulation for the shortest path problem appears in A (otherwise, see [20] Section 5.4 for example). If we employ a primal formulation for the shortest path problem, then the obtained formulation for the least core problem includes non-linear terms, which causes some difficulties (see A for details).

We introduce a vector of variables $(y(v) \mid v \in V)$ and an artificial variable $y_{T}$. Then, we have a linear programming formulation of the shortest path problem:

$$
\begin{aligned}
& \mathrm{D}(\boldsymbol{x}): \max \cdot y_{T}-y(s), \\
& \text { s.t. } y(v)-y(u) \leq 0 \quad\left(\forall(u, v) \in A_{0}\right) \text {, } \\
& y(v)-y(u) \leq x_{i} \quad\left(\forall i \in N, \forall(u, v) \in A_{i}\right), \\
& y(v)=y_{T} \quad(\forall v \in T) .
\end{aligned}
$$

The last constraint $y(v)=y_{T}(\forall v \in T)$ corresponds to a contraction operation of the set of vertices $T$ to an artificial terminal vertex.

Since problem $\mathrm{D}(\boldsymbol{x})$ is a maximization problem, the length of the $s-T$ shortest path is greater than or equal to $1-\varepsilon$ if and only if there exists a 
feasible solution of $\mathrm{D}(\boldsymbol{x})$ satisfying $y_{T}-y(s) \geq 1-\varepsilon$. Thus, $\boldsymbol{x} \in \mathbb{R}^{N}$ is in the least core if and only if $\boldsymbol{x}$ is a subvector of an optimal solution to the following problem:

$$
\begin{array}{rlr}
\mathrm{P} 2: \operatorname{min.} & \varepsilon & \\
\text { s.t. } & y_{T}-y(s) \geq 1-\varepsilon, & \\
& y(v)-y(u) \leq 0 \quad & \left(\forall(u, v) \in A_{0}\right), \\
& y(v)-y(u) \leq x_{i} & \left(\forall i \in N, \forall(u, v) \in A_{i}\right), \\
& y(v)=y_{T} & (\forall v \in T), \\
& \sum_{i \in N} x_{i}=1, & \\
& x_{i} \geq 0 &
\end{array}
$$

where $\varepsilon, y_{T},\{y(v) \mid v \in V\}$ and $\left\{x_{i} \mid i \in N\right\}$ are continuous variables. Here we note that $\boldsymbol{x}$ is a fixed vector in $\mathrm{D}(\boldsymbol{x})$ and a vector of variables in P2.

The numbers of variables and constraints in $\mathrm{P} 2$ are bounded by $\mathrm{O}\left(n W_{+}\right)$. Thus, a polynomial time algorithm for general linear programming problems solves P2 and finds a payoff vector in the least core in pseudo polynomial time on the input size of the integer vector $\left[q ; w_{1}, w_{2}, \ldots, w_{n}\right]$.

In the following part, we summarize known results related to the behavior of $W_{+}=\sum_{i=1}^{n} w_{i}$. When a given simple game $(N, v)$ is a weighted voting game, the corresponding integer-weights representation $\left[q ; w_{1}, w_{2}, \ldots, w_{n}\right]$ is not unique. Moreover, Isbell [21] found an example of a weighted simple game with 12 players without a unique minimum-sum integer-weight representation. Examples for 9, 10, or 11 players are given in Freixas and Molinero [22, 23. The problem of determining a minimum-sum integerweight representation is computationally difficult, as the problem of deciding a dummy player is NP-complete [7, 9] and a voting weight of every dummy player is equal to zero in a minimum-sum integer-weight representation. In cases of $n \leq 9$ players, Kurz [24] enumerated (minimal) integerweight representations. In general, Kawana and Matsui [25] showed that every weighted voting game has an integer-weight representation satisfying $W_{+} \leq(n+2)^{\frac{n+2}{2}}(1 / 2)^{n}-1$. In the next section, we describe two practical examples satisfying $\left(n, W_{+}\right)=(51,538),(27,345)$. 


\section{Computational Experiences}

This section reports the results of our numerical experiments. All the experiments were conducted on a PC running the Windows 10 Pro operating system with an Intel(R) Core(TM) i7-8700@3.2GHz processor and 16 GB RAM. All instances were solved using CPLEX 12.8.0.0 implemented in Python 3.5.4.

A weighted voting game reported in [26] (Section 12.4) for a voting process in the United States has a quota and a vector of weights

$$
\begin{aligned}
& {[270 ; 45,41,27,26,26,25,21,17,17,14,13,13,12,12,12,11, \underbrace{10, \ldots, 10}_{4 \text { times }}, \underbrace{9, \ldots, 9}_{4 \text { times }},} \\
& 8,8, \underbrace{7, \ldots, 7}_{4 \text { times }}, \underbrace{6, \ldots, 6}_{4 \text { times }}, 5, \underbrace{4, \ldots, 4}_{9 \text { times }}, \underbrace{3, \ldots, 3}_{7 \text { times }}], \text { where } n=51 \text { and } W_{+}=538 .
\end{aligned}
$$

We used the above data and solved problem P2. The computational time is 1.343 seconds and the optimal value $\varepsilon^{*}$ is equal to $0.49814 \cdots$. The obtained vector in the least core is proportional to the vector of voting weights to ensure that the sum is equal to 1.

A game of the power of countries in the EU Council [27, 28] with 27 players is defined by

$$
\begin{array}{r}
{[255 ; 29,29,29,29,27,27,14,13,12,12,12,12,12,10,10,10} \\
7,7,7,7,7,4,4,4,4,4,3], \text { where } n=27 \text { and } W_{+}=345 .
\end{array}
$$

In this case, the computational time for solving P2 is 0.063 seconds and the optimal value $\varepsilon^{*}$ is equal to $0.26086 \cdots$. As in the case of the voting process in the United States, the obtained vector in the least core is proportional to the vector of voting weights. Here we note that a vector in the least core is not necessarily unique, and thus vectors "obtained" by solving P2 are proportional to the voting weights in the above two cases.

We generated random artificial instances as follows. For each pair $(n, U) \in$ $\{40,60,80,100,120\}^{2}$, we constructed 20 weighted voting games by generating voting weight $w_{i}(i \in\{1,2, \ldots, n\})$ uniformly at random from the set of integers $\{1,2, \ldots, U\}$. We set $q$ to an integer obtained by rounding $0.9 W_{+}=0.9 \sum_{i=1}^{n} w_{i}$. Table 1 shows the average of computation time of 20 instances generated for each cell. We applied the least-squares regression method to $5 \cdot 5 \cdot 20=500$ instances. The estimated regression equation is as follows:

$$
\ln (\text { comp. time }[\mathrm{s}])=-25.208+2.885 \ln n+1.891 \ln W_{+} .
$$




\begin{tabular}{c|rrrrr}
\multirow{2}{*}{$\begin{array}{c}\text { Table 1: Computation time }[\mathrm{s}] . \\
U\end{array}$} & \multicolumn{5}{c}{$n$ (number of players) } \\
\cline { 2 - 6 }$\left(\mathrm{UB}\right.$ of $\left.w_{i}\right)$ & 40 & 60 & 80 & 100 & 120 \\
\hline 40 & 0.2 & 1.1 & 3.6 & 9.7 & 20.4 \\
60 & 0.4 & 2.3 & 9.2 & 23.0 & 47.9 \\
80 & 0.5 & 3.9 & 14.7 & 42.9 & 91.9 \\
100 & 0.6 & 5.5 & 23.9 & 68.7 & 198.5 \\
120 & 0.8 & 7.6 & 35.6 & 121.1 & 280.7
\end{tabular}

We give an additional experiment for checking whether a vector in the least core is proportional to a given vector of voting weights. In general, this property does not hold. We need only recall examples of dummy players with positive voting weights. Our experiences are limited to randomly generated artificial problems with small numbers of players. We set the number of players at $n \in\{8,10,12,15,18\}$ and generated 100 vectors of weights for each $n$. A voting weight $w_{i}$ for each player $i$ is chosen uniformly at random from the set of integers $\{1,2, \ldots, 20\}$. For each generated vector of weights, we tested all the cases in which a quota $q$ is equal to an integer satisfying $(1 / 4) W_{+} \leq q \leq(3 / 4) W_{+}$. Table 2 shows the results of our experiment. The rows denoted by "\# VLC || weights" (and "\# VLC X weights") show the number of instances satisfying the condition that the obtained vector in the least core is (not) proportional to the voting weights, respectively. The row denoted by "ratio [\%]" shows the ratio of the number of instances in the row "\# VLC || weights" to the total number of generated instances. When $n=18$, all the generated instances satisfy the condition that the obtained vector in the least core is proportional to the voting weights. Our results indicate a property of the nucleolus of weighted voting games shown by Kurz, Napel, and Nohn [29], who proved that under some assumptions, when the relative weight of every player tends to zero, the nucleolus converges to a vector proportional to the voting weights.

\section{$5 \quad$ Vector Weighted Voting Games}

In this section, we discuss a case of vector weighted voting games, briefly. Chalkiadakis, Elkind, and Wooldridge [14] summarized previous works re- 
Table 2: A payoff vector in the least core and voting weights.

\begin{tabular}{r|rrrrrr} 
\# of players & 5 & 8 & 10 & 12 & 15 & 18 \\
\hline \# VLC || weights & 46 & 89 & 1088 & 4182 & 7251 & 9060 \\
\# VLC X weights & 2490 & 3792 & 3987 & 1833 & 167 & 0 \\
\hline ratio [\%] & 1.81 & 2.29 & 21.44 & 69.53 & 97.75 & 100
\end{tabular}

lated to vector weighted voting games.

Let $k$ be a given positive integer. For any $k^{\prime} \in\{1,2, \ldots, k\}$, let $G_{k^{\prime}}=$ $\left[q_{k^{\prime}} ; w_{k^{\prime} 1}, w_{k^{\prime} 2}, \ldots, w_{k^{\prime} n}\right]$ be a weighted voting game defined on a mutual set of players $N=\{1,2, \ldots, n\}$. The corresponding sets of winning coalitions are denoted by $\mathcal{W}_{k^{\prime}}\left(k^{\prime} \in\{1,2, \ldots, k\}\right)$. An intersection of $k$ games $G_{1}, G_{2}, \ldots, G_{k}$ is a simple game $\left(N, v_{\wedge}\right)$ defined by a characteristic function $v_{\wedge}: 2^{N} \rightarrow\{0,1\}$ satisfying $v_{\wedge}(S)=1$ if and only if $S \in \mathcal{W}_{k^{\prime}}\left(\forall k^{\prime} \in\right.$ $\{1,2, \ldots, k\})$. The dimensionality, $k$, of a $k$-vector weighted voting game can be understood as a measure of the complexity of the game. Elkind et al. 30] showed that the problems of 'equivalence' and 'minimality' of $k$-vector weighted voting games are computationally difficult. They also provided efficient algorithms for those cases where two conditions are met: $k$ is small and the weights are polynomially bounded.

For calculating a payoff vector in the least core of $\left(N, v_{\wedge}\right)$, we only need to change the definition of the directed graph $\Gamma=(V, A)$ as follows. We introduce a vertex set $V=[n]_{0} \times\left[W_{1+}\right]_{0} \times \cdots \times\left[W_{k+}\right]_{0}$ where $W_{k^{\prime}+}=\sum_{i=1}^{n} w_{k^{\prime} i}$ $\left(k^{\prime} \in\{1,2, \ldots, k\}\right)$. A vertex in $V$ is denoted by $(i, \boldsymbol{\alpha})=\left(i, \alpha_{1}, \alpha_{2}, \ldots, \alpha_{k}\right) \in$ $\mathbb{Z}^{k+1}$. For each player $i \in N$, we denote the vector $\left(w_{1 i}, w_{2 i}, \ldots, w_{k i}\right)$ by $\boldsymbol{w}_{* i}$. An arc set $A=A_{0} \cup A_{1} \cup \cdots \cup A_{n}$ is defined by

$$
\begin{aligned}
& A_{0}=\left\{((i-1, \boldsymbol{\alpha}),(i, \boldsymbol{\alpha})) \mid i \in[n], \boldsymbol{\alpha} \in\left[W_{1+}\right]_{0} \times \cdots \times\left[W_{k+}\right]_{0}\right\}, \\
& A_{i}=\left\{\left((i-1, \boldsymbol{\alpha}),\left(i, \boldsymbol{\alpha}+\boldsymbol{w}_{* i}\right)\right) \mid(i-1, \boldsymbol{\alpha}) \in V \text { and }\left(i, \boldsymbol{\alpha}+\boldsymbol{w}_{* i}\right) \in V\right\}
\end{aligned}
$$

for each $i \in N=[n]$. We also set $T=\left\{(n, \boldsymbol{\alpha}) \subseteq V \mid q_{k^{\prime}} \leq \alpha_{k^{\prime}} \quad\left(\forall k^{\prime} \in\right.\right.$ $\{1,2, \ldots, k\})\}$. Then, it is easy to see that problem P2 finds a payoff vector in the least core of a simple game $\left(N, v_{\wedge}\right)$. The numbers of variables and constraints of P2 are bounded by $O\left(n \prod_{k^{\prime}=1}^{k} W_{k^{\prime}+}\right)$ and thus P2 remains a pseudo polynomial of size LP.

A union of $k$ games $G_{1}, G_{2}, \ldots, G_{k}$ is a simple game $\left(N, v_{\vee}\right)$ defined by a characteristic function $v_{\vee}: 2^{N} \rightarrow\{0,1\}$ satisfying $v_{\vee}(S)=1$ if and only 
if $\exists k^{\prime} \in\{1,2, \ldots, k\}, S \in \mathcal{W}_{k^{\prime}}$. We can deal with this case by setting $T=$ $\left\{(n, \boldsymbol{\alpha}) \subseteq V \mid \exists k^{\prime} \in\{1,2, \ldots, k\}, q_{k^{\prime}} \leq \alpha_{k^{\prime}}\right\}$.

It is easy to see that we can extend our formulation to a Boolean weighted voting game [31, 32] obtained by combining some weighted voting games according to a complex Boolean formula.

\section{Conclusion}

In this paper, we proposed a pseudo polynomial size linear programming formulation for calculating a payoff vector in the least core of a weighted voting game. When we employ our formulation, a commercial solver finds a payoff vector in the least core of practical weighted voting games in a few seconds. Our computational experiences indicate that a vector in the least core is proportional to the voting weights in many cases.

We also extended our formulation to vector weighted voting games. Further research is needed to evaluate the computational performance of the formulation for vector weighted voting games.

\section{A Primal Formulation of Shortest Path Prob- lem}

In this section, we describe an ordinary primal formulation of the $s$ - $T$ shortest path defined on the acyclic directed graph $\Gamma=(V, A)$ with arc length function $w^{\boldsymbol{x}}: A \rightarrow \mathbb{R}$. First, we transform the problem into an ordinary 2-terminal shortest path problem by introducing an artificial terminal vertex $t^{\bullet}$ and artificial $\operatorname{arcs} A^{\bullet}=T \times\left\{t^{\bullet}\right\}$ with 0-length. We denote the obtained acyclic directed graph by $\Gamma^{\bullet}=\left(V \cup\left\{t^{\bullet}\right\}, A \cup A^{\bullet}\right)$. For each vertex $v \in V \cup\left\{t^{\bullet}\right\}$, we denote by $\delta^{+}(v)$ and $\delta^{-}(v)$ the set of outgoing and incoming arcs of vertex $v$ in $\Gamma^{\bullet}$. For each arc $a \in A \cup A^{\bullet}$, we introduce a variable $\xi(a)$ that takes the value 1 if the arc $a$ belongs to a shortest path. A standard primal linear programming formulation to determine a shortest path from $s$ to $t^{\bullet}$ on $\Gamma^{\bullet}$ is 
the following:

$$
\begin{aligned}
& \mathrm{P}(\boldsymbol{x}): \min . \sum_{i=1}^{n} \sum_{a \in A_{i}} x_{i} \xi(a), \\
& \text { s.t. } \sum_{a \in \delta^{+}(v)} \xi(a)-\sum_{a \in \delta^{-}(v)} \xi(a)= \begin{cases}1 & (v=s), \\
-1 & \left(v=t^{\bullet}\right), \\
0 & (\text { otherwise }), \\
\xi(a) \geq 0 & (\forall a \in A) .\end{cases}
\end{aligned}
$$

Problem $\mathrm{D}(\boldsymbol{x})$ defined in Section 3, is obtained from the dual of $\mathrm{P}(\boldsymbol{x})$ by trivial transformations.

By setting the length of the $s$ - $t^{\bullet}$ shortest path on $\Gamma^{\bullet}$ to $1-\varepsilon, \boldsymbol{x} \in \mathbb{R}^{N}$ is in the least core if and only if $\boldsymbol{x}$ is a subvector of an optimal solution to the following problem:

$$
\begin{aligned}
\mathrm{P}^{\prime}: \min . & \varepsilon \\
\text { s.t. } & 1-\varepsilon=z(\boldsymbol{x}) \\
& \sum_{i \in N} x_{i}=1, \\
& x_{i} \geq 0 \quad(\forall i \in N),
\end{aligned}
$$

where $z(\boldsymbol{x})$ denotes the optimal value of $\mathrm{P}(\boldsymbol{x})$. The above formulation implicitly includes nonlinear terms $x_{i} \xi(a)$, which are difficult to address directly. The problem $\mathrm{P}^{\prime}$ corresponds to the shortest path problem with variable arc length, where related problems are discussed in [33, 34] for example.

\section{References}

[1] L. S. Shapley, M. Shubik, A method for evaluating the distribution of power in a committee system, The American Political Science Review 48 (3) (1954) 787-792.

[2] J. F. Banzhaf III, Weighted voting doesn't work: A mathematical analysis, Rutgers Law Review 19 (1964) 317-343.

[3] J. Deegan, E. W. Packel, A new index of power for simple $n$-person games, International Journal of Game Theory 7 (2) (1978) 113-123. 
[4] T. Matsui, Y. Matsui, A survey of algorithms for calculating power indices of weighted majority games, Journal of the Operations Research Society of Japan 43 (1) (2000) 71-86.

[5] X. Deng, C. H. Papadimitriou, On the complexity of cooperative solution concepts, Mathematics of Operations Research 19 (2) (1994) 257-266.

[6] E. Elkind, L. A. Goldberg, P. W. Goldberg, M. Wooldridge, On the computational complexity of weighted voting games, Annals of Mathematics and Artificial Intelligence 56 (2) (2009) 109-131.

[7] M. R. Garey, D. S. Johnson, Computers and Intractability, Vol. 174, Freeman San Francisco, 1979.

[8] Y. Matsui, T. Matsui, NP-completeness for calculating power indices of weighted majority games, Theoretical Computer Science 263 (1-2) (2001) 305-310.

[9] K. Prasad, J. S. Kelly, NP-completeness of some problems concerning voting games, International Journal of Game Theory 19 (1) (1990) 1-9.

[10] M. Maschler, B. Peleg, L. S. Shapley, Geometric properties of the kernel, nucleolus, and related solution concepts, Mathematics of Operations Research 4 (4) (1979) 303-338.

[11] D. Schmeidler, The nucleolus of a characteristic function game, SIAM Journal on Applied Mathematics 17 (6) (1969) 1163-1170.

[12] E. Elkind, D. Pasechnik, Computing the nucleolus of weighted voting games, in: Proceedings of the Twentieth Annual ACM-SIAM Symposium on Discrete Algorithms, SIAM, 2009, pp. 327-335.

[13] K. Pashkovich, Computing the nucleolus of weighted voting games in pseudo-polynomial time, arXiv preprint arXiv:1810.02670.

[14] G. Chalkiadakis, E. Elkind, M. Wooldridge, Computational Aspects of Cooperative Game Theory, Morgan \& Claypool Publishers, 2011.

[15] G. Dantzig, Linear Programming and Extensions, Princeton University Press, 1963. 
[16] L. G. Khachiyan, Polynomial algorithms in linear programming, USSR Computational Mathematics and Mathematical Physics 20 (1) (1980) 53-72.

[17] R. G. Bland, D. Goldfarb, M. J. Todd, The ellipsoid method: A survey, Operations Research 29 (6) (1981) 1039-1091.

[18] M. Grötschel, L. Lovász, A. Schrijver, Geometric Algorithms and Combinatorial Optimization, Springer, 1987.

[19] N. Karmarkar, A new polynomial-time algorithm for linear programming, in: Proceedings of the Sixteenth Annual ACM Symposium on Theory of Computing, 1984, pp. 302-311.

[20] C. H. Papadimitriou, K. Steiglitz, Combinatorial Optimization: Algorithms and Complexity, Prentice Hall, 1981.

[21] J. R. Isbell, A class of majority games, The Quarterly Journal of Mathematics 7 (1) (1956) 183-187.

[22] J. Freixas, X. Molinero, On the existence of a minimum integer representation for weighted voting systems, Annals of Operations Research 166 (1) (2009) 243.

[23] J. Freixas, X. Molinero, Weighted games without a unique minimal representation in integers, Optimisation Methods \& Software 25 (2) (2010) 203-215.

[24] S. Kurz, On minimum sum representations for weighted voting games, Annals of Operations Research 196 (1) (2012) 361-369.

[25] A. Kawana, T. Matsui, Trading transforms of non-weighted simple games and integer weights of weighted simple games, Theory and Decisiondoi:https://doi.org/10.1007/s11238-021-09831-2.

[26] G. Owen, Game Theory, Emerald Group Publishing Limited, 1995.

[27] D. S. Felsenthal, M. Machover, The treaty of nice and qualified majority voting, Social Choice and Welfare 18 (3) (2001) 431-464. 
[28] J. M. Bilbao, J. R. Fernandez, N. Jiménez, J. J. Lopez, Voting power in the European Union enlargement, European Journal of Operational Research 143 (1) (2002) 181-196.

[29] S. Kurz, S. Napel, A. Nohn, The nucleolus of large majority games, Economics Letters 123 (2) (2014) 139-143.

[30] E. Elkind, L. A. Goldberg, P. W. Goldberg, M. J. Wooldridge, On the dimensionality of voting games., in: Proceedings of the Twenty-Third AAAI Conference on Artificial Intelligence, 2008, pp. 69-74.

[31] P. Faliszewski, E. Elkind, M. Wooldridge, Boolean combinations of weighted voting games, in: Proceedings of The 8th International Conference on Autonomous Agents and Multiagent Systems-Volume 1, 2009, pp. $185-192$.

[32] S. Kurz, S. Napel, Dimension of the Lisbon voting rules in the EU Council: a challenge and new world record, Optimization Letters 10 (6) (2016) 1245-1256.

[33] T. L. Magnanti, P. Mirchandani, Shortest paths, network design and associated polyhedra, Operations Research Center Working Paper OR 215-90.

[34] P. Cappanera, M. P. Scaparra, Optimal allocation of protective resources in shortest-path networks, Transportation Science 45 (1) (2011) 64-80. 